\title{
Safe and Complete Contig Assembly Through Omnitigs
}

\section{Tomescu, Alexandru I.}

2017-06-01

Tomescu , A I \& Medvedev , P 2017 , ' Safe and Complete Contig Assembly Through

Omnitigs ' , Journal of Computational Biology , vol. 24 , no. 6 , pp. 590-602 . https://doi.org/10.1089/cmb.2016.0141

http://hdl.handle.net/10138/313118

https://doi.org/10.1089/cmb.2016.0141

acceptedVersion

Downloaded from Helda, University of Helsinki institutional repository.

This is an electronic reprint of the original article.

This reprint may differ from the original in pagination and typographic detail.

Please cite the original version. 


\title{
Safe and complete contig assembly via omnitigs
}

\author{
Alexandru I. Tomescu ${ }^{1}$ and Paul Medvedev2;3;4 \\ ${ }^{1}$ Helsinki Institute for Information Technology HIIT, \\ Department of Computer Science, University of Helsinki \\ P.O. Box 68, FI-00014, Helsinki, Finland \\ E-mail: tonescu@s. hel si nki .fi , Fax: +3589 8764314 \\ ${ }^{2}$ Department of Computer Science and Engineering, The Pennsylvania State University, USA \\ ${ }^{3}$ Department of Biochemistry and Molecular Biology, The Pennsylvania State University, USA \\ ${ }^{4}$ Genome Sciences Institute of the Huck, The Pennsylvania State University, USA
}

\begin{abstract}
A bstract. Contig assembly is the rst stage that most assemblers solve when reconstructing a genome from a set of reads. Its output consists of contigs $\{$ a set of strings that are promised to appear in any genome that could have generated the reads. From the introduction of contigs 20 years ago, assemblers have tried to obtain longer and longer contigs, but the following question remains: given a genome graph G (eg. a de Bruijn, or a string graph), what are all the strings that can be safely reported from $G$ as contigs? In this paper we answer this question using a model where the genome is a circular covering walk. We also give a polynomial time algorithm to nd such strings, which we call omnitigs. Our experiments show that omnitigs are $66 \%$ to $82 \%$ longer on average than the popular unitigs, and $29 \%$ of dbSNP locations have more neighbors in omnitigs than in unitigs.
\end{abstract}

K eywords: genome assembly, contig assembly, safe and complete algorithm, graph algorithm, omnitig

A preliminary version of this paper appeared in RECOMB 2016 
\begin{tabular}{|l|l|l|l|}
\hline & & &
\end{tabular} 
with previous papers that developed better theoretical underpinnings [31,10,26,23], it is necessary to prove results in a somewhat idealized setting. While this paper falls short of analyzing real data, we believe that omnitigs can be incorporated into practical genome analysis and assembly tools \{ similar to the way that error-free studies of de Bruijn [31] and paired de Bruijn graphs [23] became the basis of practical assemblers $[32,40,1]$.

\section{Related work}

The number of related assembly papers is vast, and we refer the reader to some surveys [24,28]. For an empirical evaluation of the correctness of several state-of-the-art assemblers, see [36]. Here, we discuss work on the theoretical underpinnings of assembly.

There are many formulations of the genome assembly problem. One of the rst asks to reconstruct the genome as a shortest superstring of the reads [30,16,15]. Later formulations referred to a graph built from the reads, such as a de Bruijn graph [10,32] or a string graph [26,38]. In an (edgecentric) de Bruijn graph, the reconstructed genome can be modeled as a circular walk covering every edge exactly once| Eulerian [32] or at least once| a Chinese Postman tour [21,22,27,13]. In a string graph, the reconstructed genome can be modeled as a circular walk covering every node exactly once Hamiltonian| [11,29], or at least once [27]. These models have also been considered in their weighted versions [21,27,29], or augmented to include other information, such as matepairs [33/23]14]. Each such notion of genomic reconstruction brought along questions concerning its validity. For example, under which conditions on the sequencing data (e.g., coverage, read length, error rate) is there at least one reconstruction [19/25], or exactly one reconstruction [5/18 32]. If there are many possible reconstructions, then what is their number [17/8] and in which aspects one is di erent from all others [8]. In contrast to the framework of this paper, most of these formulations deal with nding a single genomic reconstruction as opposed to a set of safe strings (i.e. contigs).

There are a few notable exceptions. In [4], Boisvert and colleagues also de ne the assembly problem in terms of nding contigs, rather than a single reconstruction. Nagarajan and Pop [27] observe that Waterman's characterization [41] of the graphs with a unique Eulerian tour leads to a simpleal gorithm for nding all safestrings when a genomic reconstruction is an Eulerian tour. They al so suggest an approach for nding all the safe strings when a genomic reconstruction is a Chinese Postman tour [27]. We note, however, that in the Eulerian model, the exact copy count of each edge should be known in advance, while in the Chinese Postman model (minimizing the length of the genomic reconstruction), the solution will over-collapse all tandem repeats. Furthermore, these approaches have not been implemented and hence their e ectiveness is unknown.

In practice, the most commonly employed safe strings are the ones spelled by maximal unitigs, where unitigs are paths whose internal nodes have in- and out-degree one. Figure 2 shows an example of the output of the unitig algorithm, and also illustrates that it does not identify all safe strings. The EULER assembler [32] takes unitigs a step further and identi es strings spelled by paths whose internal nodes have out-degree equal to one (with no constraint on their in-degre). It can be shown that such strings are also safe. However, the most complete characterization of safe strings that we found is given by the $Y$-to- $V$ algorithm [2212]17]. Consider a node $v$ with exactly one in-neighbor $\mathrm{u}$ and more than one out-neighbors $\mathrm{w}_{1} ;:: ; ; \mathrm{w}_{\mathrm{d}}$. The $\mathrm{Y}$-to- $\mathrm{V}$ reduction applied to $\mathrm{v}$ removes $v$ and its incident edges from the graph and adds nodes $v_{1} ;:: ; ; v_{d}$ with edges from $u$ to $v_{i}$ and from $v_{i}$ to $w_{i}$, for all 16 i $6 \mathrm{~d}$. The $Y$-to- $V$ reduction is de ned symmetrically for nodes with out-degree exactly one and in-degree greater than one. Figure 1 illustrates the de nition. The 


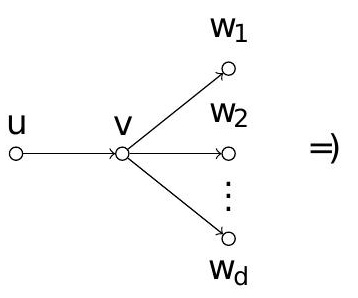

(a)

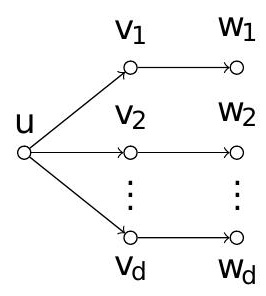

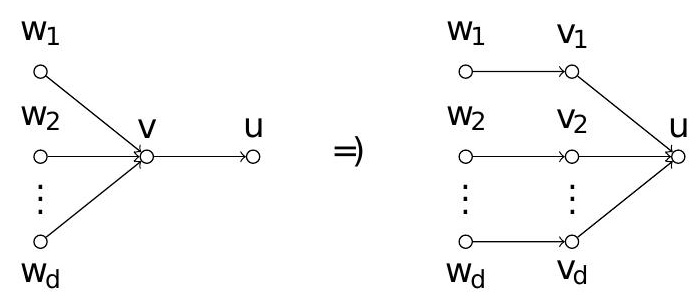

(b)

Fig. 1. The Y-to-V reduction applied to node v. In Fig. 1(b) $v$ has in-degree exactly one; in Fig. 1(b) $v$ has out-degree exactly one.

$\mathrm{Y}$-to-V algorithm proceeds by repeatedly applying $\mathrm{Y}$-to-V reductions, in arbitrary order, for as long as possible. The al gorithm then outputs the strings spelled by the maximal unitigs in the nal graph (see Figure2 $2 \mathrm{~d}$ for an example). The $\mathrm{Y}$-to-V algorithm can also be shown to be safe, but, as we will show in Figure 2, it does not always output all the safe strings. We are not aware of any study that compares the merits of $\mathrm{Y}$-to-V contigs to unitigs, and we therefore perform this analysis in Section 8,

\section{Basic de nitions}

Given a string $x$ and an index 16 i 6 jxj, we de ne pre $(x ; i)$ and $\operatorname{suf}(x ; i)$ as its length $i$ pre $x$ and su $x$, respectively. If $x$ and $y$ are two strings, and suf $(x ; k)=\operatorname{pre}(y ; k)$ for some $k 6$ jxj 1 , then we de ne $x \quad k y$ as $x[1:: j x j \quad k]$ concatenated with $y$. This captures the notion of merging two overlapping strings. A k-mer of $x$ is a substring of length $k$. Let $R$ be a set of strings, which we equivalently refer to as reads. The node-centric de Bruijn graph built on $R$, denoted $D B_{n c}^{k}(R)$, is the graph whose set of nodes is the set of all $k$-mers of $R$, in which there is an edge from a node $x$ to a node y $\mathrm{i} \operatorname{suf}(x ; k \quad 1)=\operatorname{pre}(y ; k \quad 1)$ [7]. The edgecentric de Bruijn graph built on $R$, denoted $D B_{e c}^{k}(R)$ is de ned similarly to $D B_{n c}^{k}(R)$, with the di erence that there is an edge from $x$ to $y i$ $\operatorname{suf}(x ; k \quad 1)=\operatorname{pre}(y ; k \quad 1)$ and $x \quad{ }^{k}{ }^{1} y$ is a substring of some string in $R$ [10]. The weight of the edges of $D B_{n c}^{k}(R)$ and $D B_{e c}^{k}(R)$ is $k 1$.

Let $G$ be a graph, possibly with paralle edges and seff-loops. The number of nodes and edges in a graph are denoted by $n$ and $m$, respectively. We use $N(v)$ to denote the set of inneighbors and $\mathrm{N}^{+}(\mathrm{v})$ to denote the set of out-neighbors of a node $\mathrm{v}$. A walk $\mathrm{w}$ is a sequence $\left(v_{0} ; e_{0} ; v_{1} ; e_{1} ;:: ; v_{t} ; e_{i} ; v_{t+1}\right)$ where $v_{0} ;:: ; v_{t+1}$ are nodes, and each $e$ is an edge from $v_{i}$ to $v_{i+1}$, and $t>1$. Its length is its number of edges, namely $t+1$. A path is a walk where the nodes are all distinct, except possibly the rst and last nodes may be the same, in which case it will also be called a cycle. Walks and paths of length at least one are called proper. A walk whose rst and last nodes coincide is called circular walk. A path (walk) with rst node $u$ and last node $v$ will be called a path (walk) from u to $v$, and denoted as u-v path (walk). A walk is called node-covering if it passes through each node of $G$, and edge covering if it passes through each edge of $G$. The notions of pre $x$ and subwalk are de ned for walks in the natural way, e.g., by interpreting a walk to be a string made up by concatenating its edges. In particular, we say that a walk $w_{1}$ is a subwalk of a circular walk $w_{2}$ if $w_{1}$ interpreted as string is a substring of $w_{2}$ interpreted as circular string. In this paper we allow strings and walks to have overlapping extremities when viewed as substrings of 
a circular string, i.e., when aligned to a circular string (see e.g. the two omnitigs from Figure 2(f) which have an overlapping tail and head).

Let ' be a function labeling the nodes of $G$ and let $c$ be a function giving weights to the edges (intuitively, c should represent the length of overlaps). One can apply the notion of string spelled by a walk $\mathrm{w}=\left(\mathrm{v}_{0} ; e_{0} ; \mathrm{v}_{1} ; \mathrm{e}_{1} ;::: ; \mathrm{v}_{\mathrm{t}} ; \mathrm{e}_{\mathrm{t}} ; \mathrm{v}_{\mathrm{t}+1}\right)$ by de ning the string spelled by $\mathrm{w}$ as $\mathrm{spell}(\mathrm{w})=$ ' $\left(v_{0}\right) \quad c\left(e_{0}\right)$ ' $\left(v_{1}\right) \quad c\left(e_{1}\right) \quad c\left(e_{t}\right)$ ' $\left(v_{t+1}\right)$. When the walk $w$ is circular (thus $\left.v_{t+1}=v_{0}\right)$, then spell $(w)$ will be interpreted as the circular string obtained by overlapping the strings ' $\left(v_{0}\right)$ and ' $\left(v_{t+1}\right)$.

\section{Problem formulation}

There are various theoretical approaches to formulating the assembly problem. Here, we adopt a model that captures the most popular ones: the node-centric de Bruijn graph, the edgecentric de Bruijn graph, and the string graph [26]. We generalize these using the notion of a genome graph:

De nition 1 (Genome graph). A graph $G$ with edge-weights given by $c$ and nodelabels is a genome graph if and only if (1) for every edge $e=(u ; v)$, suf(u;c(e)) = pre(v;c(e)), and (2) for any two walks $w_{1}$ and $w_{2}, w_{1}$ is a subwalk of $w_{2}$ if and only if spell $\left(w_{1}\right)$ is a substring of $\operatorname{spell}\left(w_{2}\right)$.

Both node and edge-centric de Bruijn graphs are genome graphs, directly by their de nition. Similarly, the interested reader can verify that string graphs, as commonly de ned in [26272237], are genome graphs. Intuitively, the rst condition states that the edge-weights represent the length of overlaps between strings, while the second condition prohibits a certain redundancy in the graph. It can be broken if, for example, thereare nodes with duplicatelabels, or if somelabels are substrings of others. Or, for strings graphs, it can be broken if transitive edges are not removed from the graph [26]. We now augment a genome graph with a rule de ning a \genomic reconstruction."

De nition 2 ( $G$ raph model). A graph model $G$ is de ned by

An algorithm that transforms a set of reads $R$ into a genome graph, denoted by $G(R)$.

A rule that determines whether a walk in $G(R)$ is a genomic reconstruction.

Intuitively, a genomic reconstruction spells a genome that could have generated the observed set of reads $\mathrm{R}$. In this paper, we consider two graph models. In the edge-centric model, a genomic reconstruction is a circular edge-covering walk; its underlying genome graph can be e.g. an edgecentric de Bruijn graph. In the node-centric model, a genomic reconstruction is a circular nodecovering walk; its underlying genome graph can be a node-centric de Bruijn graph or a string graph. As mentioned in the introduction, we assume, without al ways explicitly stating it onwards, that $G(R)$ contains at least one genomic reconstruction, and for technical reasons| see the proof of Lemma 1] that $G(R)$ is always di erent from a single cycle. In fact, in this latter case the assembly problem is trivial.

We now de ne the strings that belong to all genomic reconstructions.

De nition 3 (Safe string). Given a set of reads $R$ and a graph model $G$, a string s is said to be a safe string for $G(R)$ if for every genomic reconstruction $w$ of $G(R)$, s is a substring of $s p e l(w)$.

In particular, for a nodecentric (respectively, edge-centric) graph model $G$, a string $s$ is safe if for every circular nodecovering (respectively, edge-covering) walk w, s is a substring of spell(w). It also follows from the de nitions (again assuming no gaps in coverage and no errors in the reads) 


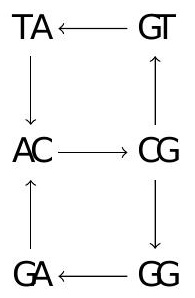

(a) Graph G

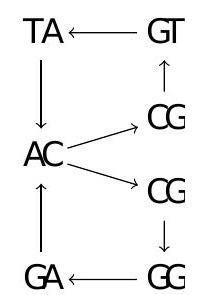

(b) Transformed graph $\mathrm{G}^{\top}$

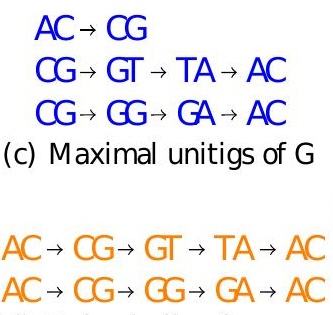

(d) Maimal aitgsof

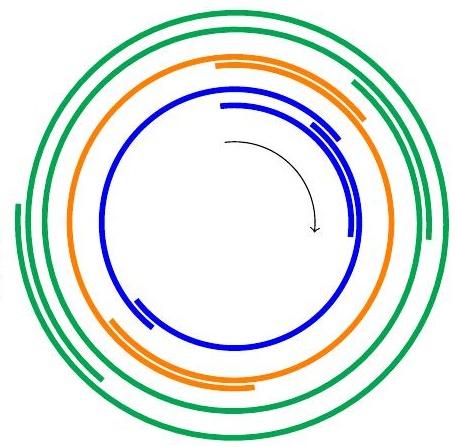

Fig. 2. The output of the three algorithms on the edgecentric de Bruijn graph $G$ from (a), built from the circular string in (f). Each contig is drawn as an arc on the wheel in (f). (c) the maximal unitigs of G; (b) the Y-to-V reduction is applied to node CG and the resulting graph $\mathrm{G}^{\top}$ is shown; no more reductions are applicable and $\mathrm{G}^{\top}$ has two maximal unitigs, shown in (d); (e) the maximal omnitigs of G; in this particular example, they are also circular edgecovering walks of $\mathrm{G}$, and one can be obtained from the other by a circular permutation. Note that this example illustrates that the $Y$-to-V algorithm does not always output all safe strings, because its output (d) does not contain the strings of (e).

that if the genome graph is $D B_{n c}^{k}(R)$ or $D B_{e c}^{k}(R)$, then a string is safe if it is a substring of every circular string with the same set of $k$-mers, or $(k+1)$-mers respectively, as $R$.

Solving the following problem gives all the information that can be safely retrieved from a graph model.

De nition 4 ( $T$ he safe and complete contig assembly problem). Given a set of reads $R$ and a graph model $G$, output all the safe strings for $G(R)$.

In this paper we solve this problem for the node and edgecentric models de ned above In Sections 5 and 6 we rst deal with the edgecentric model, and then in Section 7 we show how these results can be modi ed for the nodecentric model.

As a technical aside, our algorithms will output only maximal safe strings, in the sense that they are not a substring of any other safe string. In fact, this is desirable in practice, and moreover, the set of all safe strings is the set of all substrings of the maximal ones.

A note on assumptions: Our model makes three implicit assumptions, as outlined at the end of the Introduction. Here, we observe that such assumptions are necessary to prove even the simplest desired property: that the unitig algorithm outputs only safe strings. Let $w=\left(v_{0} ; e_{0} ; v_{1} ; e_{1} ; v_{2}\right)$ be a unitig in an edgecentric de Bruijn graph $\mathrm{G}$ built from the $(k+1)$-mers of a genome $\mathrm{S}$. If the genome is not circular (assumption (i)), then eg. the last k-mer of $S$ can be $v_{0}$, its rst k-mer can be $v_{1}$, the string $v_{0}{ }^{k} v_{1}$ can appear inside $S$, but $v_{0} \quad{ }^{k} v_{1} \quad{ }^{k} v_{2}$ does not have to appear in $S$. If there are gaps in coverage (assumption (ii)), then both an in-neighbor $v^{0}$ and an out-neighbor $v^{\boxplus}$ of $v_{1}$ may be missing from $G$ making $w$ look safe whereas in reality $v_{0}{ }^{k} v_{1} \quad k v_{2}$ may not be a substring of $\mathrm{S}$. If a read contains a sequencing error (assumption (iii)), then this creates a bubble in $\mathrm{G}$ with one of its paths being a unitig not spelling a substring of $S$. 


\section{Characterization of safe strings: omnitigs}

In this section, we provide a characterization of walks that spell safe strings (see Figure 3 for an illustration). This characterization will be the basis of our omnitig algorithm in the next section.

De nition 5 (Omnitig, edge-centric model). Let $G$ be a directed graph and let $w=$ $\left(v_{0} ; e_{0} ; v_{1} ; e_{1} ;::: ; v_{t} ; e_{i} ; v_{t+1}\right)$ be a walk in $G$. We say that $w$ is a omnitig if and only if for all 16 i $6 \mathrm{j} 6 \mathrm{t}$, there is no proper $v_{j}-v_{i}$ path with rst edge di erent from $e_{j}$, and last edge di erent frome 1 .

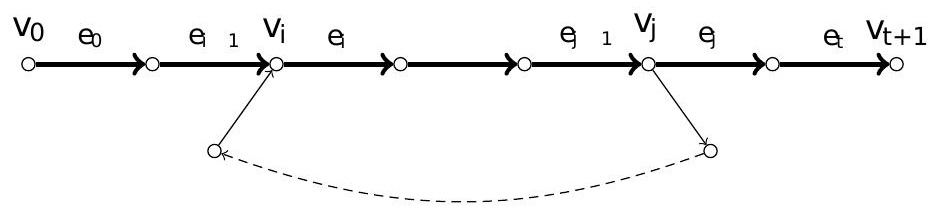

Fig. 3. An illustration of the omnitig de nition, edgecentric model

The following theorem proves that the omnitigs spell all the safe strings, using the help of an intermediary characterization of omnitigs.

Theorem 1. Given an edge-centric graph model $G=G(R)$ built for a set of reads $R$, and a string $S$, the following thre statements are equivalent:

(1) $\mathrm{S}$ is a safe string for $\mathrm{G}$;

(2) $\mathrm{S}$ is spelled by a walk $w=\left(v_{0} ; e_{0} ; v_{1} ; e_{1} ;:: ; v_{t} ; \theta_{;} ; v_{t+1}\right)$ in $G$ and $w$ is an omnitig;

(3) $\mathrm{S}$ is spelled by a walk $w=\left(v_{0} ; e_{0} ; v_{1} ; e_{1} ;:: ; v_{t} ; e_{i} ; v_{t+1}\right)$ in $G$ and for all $16 \mathrm{j} 6 \mathrm{t}$ all proper $v_{j}-v_{j}$ (circular) walks $w^{0}$ ful II at least one of the following conditions:

(i) the subwalk $\left(v_{j} ; e ;::: ; v_{t} ; e_{i} ; v_{t+1}\right)$ of $w$ is a pre $x w^{0}$, or

(ii) the subwalk $\left(v_{0} ; e_{0} ;::: ; v_{j} 1 ; e_{1} 1 ; v_{j}\right)$ of $w$ is a su $x$ of $w^{0}$, or

(iii) $\mathrm{w}$ is a subwalk of $\mathrm{w}^{\text {0. }}$

We prove Theorem 1 by proving the cyclical sequence of implications (1) ) (2) ) (3) ) (1).

Proof of (1) ) (2). Assume that $s$ is a safe string for $G$. By de nition of a genome graph, $s$ is spelled by a unique walk in $G$. Let $w=\left(v_{0} ; e_{0} ; v_{1} ; e_{1} ;:: ; v_{t} ; e_{i} ; v_{t+1}\right)$ be this walk, and let $A$ be $a$ circular edge-covering walk of $G$; thus $A$ contains $w$ as subwalk, and $s$ is a sub-string of spell(A).

Assume for a contradiction that there exist 16 i 6 j $6 \mathrm{t}$, and a proper $v_{j}-v_{i}$ path $p$ with rst edge di erent from e and last edge di erent from e 1 . From $A$, we will construct another circular edge-covering walk $B$ of $G$ which does not contain $w$ as subwalk, and hence, by the de nition of a genome graph, also spell(B) does not contain $\mathrm{S}$ as sub-string. This will contradict the safeness of s. Whenever $A$ visits node $v_{j}$, then $B$ follows the $v_{j}-v_{i}$ path $p$, then follows $\left(v_{i} ; e_{;} ;:: ; e_{j} 1 ; v_{j}\right)$, and nally continues as $A$. To see that $w$ does not appear as a subwalk of $B$, consider the subwalk $w^{0}=\left(v_{i} 1 ; e_{1} ; v_{i} ; e_{i} ;:: ; e_{1} 1 ; v_{j} ; \Theta_{i} ; v_{j+1}\right)$ of $w$ (recall that 16 i $\left.6 j 6 \mathrm{t}\right)$. Since $p$ is proper, and its rst edge is di erent from $e_{\text {and }}$ and last edge is di erent from $e_{1}$, then, by construction, the only way that $w^{0}$ can appear in $B$ is as a subwalk of $p$. However, this implies that both $v_{j}$ and $v_{i}$ appear twice on $p$, contradicting the fact that $p$ is a path. 


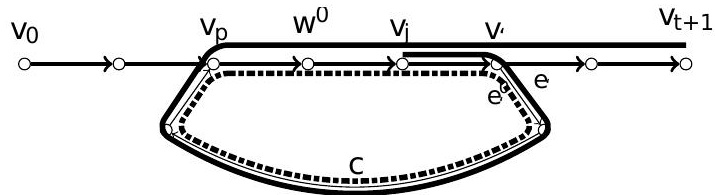

(a)

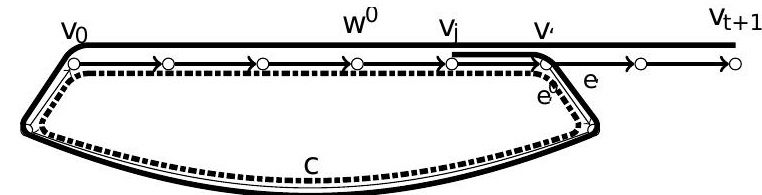

(b)

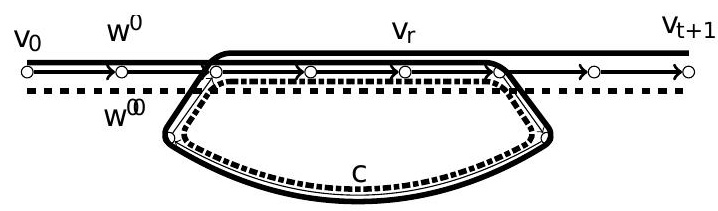

(c)

Fig. 4. Illustration of three cases in the proof of the implication (2) ) (3) of Theorem 1

Proof of (2) ) (3). Suppose that $w$ is an omnitig, and assume for a contradiction that there exists a proper $v_{j}-v_{j}$ walk (for some 16 j 6 t) not satisfying (i) $\left\{\left(\right.\right.$ iii). Let $w^{0}$ be the shortest such walk. Since $w^{0}$ does not have $\left(v_{j} ; e_{i} ;:: ; v_{t} ; e_{i} ; v_{t+1}\right)$ as pre $x$, then there exists a rst node $v$. on $w$, j 6 ' $6 \mathrm{t}$, such that from $v^{\prime}, w^{0}$ continues with an edge $e^{0} \theta$ e. Symmetrically, since $w^{0}$ does not have $\left(v_{0} ; e_{0} ;::: ; v_{j} 1 ; e_{j} 1 ; v_{j}\right)$ as su $x$, let $v_{i}$ be the last node of $w, 16$ i 6 j such that before entering $v_{i}$, the walk $w^{0}$ uses an edge $e_{1}^{0}{ }_{1} \sigma e_{1}{ }_{1}$. Let $w_{0}^{0}$ denote the subwalk of $w^{0}$ between $e^{0}$ and $\mathrm{e}^{0}{ }_{1}$ (inclusive). If $\mathrm{w}_{0}^{0}$ is a path, then $\mathrm{w}^{\infty}$ is a proper $\mathrm{v}-\mathrm{v}_{\mathrm{i}}$ path, 16 i $6^{\prime}$ ' $6 \mathrm{t}$, whose rst edge $\mathrm{e}^{0}$ is di erent from $e$, and its last edge $e_{i}^{0}{ }_{1}$ is di erent from $e_{1}{ }_{1}$, which contradicts the fact that $w$ is an omnitig. We now prove that $\mathrm{w}^{0}$ is in fact a path.

Suppose for a contradiction that it is not, thus that it contains a cycle c, with $c \in w^{0}$. Let $w^{\infty}$ be the walk obtained from $w^{0}$ by removing the cycle c. Observe that $w^{\infty}$ is still a proper $v_{j}-v_{j}$ walk. We show that $w^{\oplus}$ still does not satisfy (i) $\left\{\left(\right.\right.$ iii), which will contradict the minimality of $w^{0}$. Assume for a contradiction that $w^{\oplus}$ satis es at least one of (i), (ii), or (iii).

First, if $w^{\infty}$ satis $e s(i)$, this implies that the edge $e^{0}$ out-going from $v$. belongs to $c$, and after traversing $c$, the walk $w^{0}$ continues through $\left(v_{1} ; e ;:: ; v_{t} ; e_{i} ; v_{t+1}\right)$ (seFigures $4(a)$ and $4(b)$ ). Let $v_{p}$ be the node of w with greatest index $p 2$ f0; : : ; ' $g$ that $c$ visits with an edge $e^{0}=\left(v ; v_{p}\right)$ not on w. Such a node exists because $c$ is a cycle and it must return to $v^{\prime}$. If $p>1$ (see Figure 4(a)), then c does not satisfy (i) $\{$ (iii). Since $c$ is proper and passes through $v$, , where 16 ' $6 \mathrm{t}$, this contradicts the minimality of $w^{0}$. Therefore, $p=0$ (see Figure $4(b)$ ), and thus, the initial $v_{j}-v_{j}$ walk $w^{0}$ (containing c as subwalk) visits $\left(v_{0} ; e_{0} ;:: ; v_{v}\right)$, and then continues through $\left(v ; e ;::: ; v_{t} ; e_{i} ; v_{t+1}\right)$. This implies that $w^{0}$ contains $w$ as subwalk, which contradicts the choice of $w^{0}$.

The second case when $w^{\infty}$ satis es (ii) is entirely symmetric.

Third, assume that $w^{\infty}$ contains $w$ as subwalk. Since $w$ is not a subwalk of $w^{0}$, this implies that $\mathrm{c}$ is a proper $\mathrm{v}_{\mathrm{r}}-\mathrm{v}_{\mathrm{r}}$ walk, for some $16 \mathrm{r} 6 \mathrm{t}$, not satisfying (i) \{(iii), which again contradicts the minimality of $\mathrm{w}^{0}$ (see Figure $4(\mathrm{c})$ ). This completes the proof of (2) )

Proof of (3) ) (1). Assume w satis es (3), and let A be a circular edge-covering walk of $G$. We need to show that $w$ is a subwalk of $A$. Let $w_{j}=\left(v_{0} ; e_{0} ;:: ; v_{j} 1 ; e_{1} 1 ; v_{j}\right)$ be the longest pre $x$ of $w$ that $A$ ever traverses, ending at some $v_{j}$. Since $A$ covers all edges, then it also covers $e_{0}$, and thus $\mathrm{j}>1$. Suppose for a contradiction that $\mathrm{j} \in \mathrm{t}+1$. 


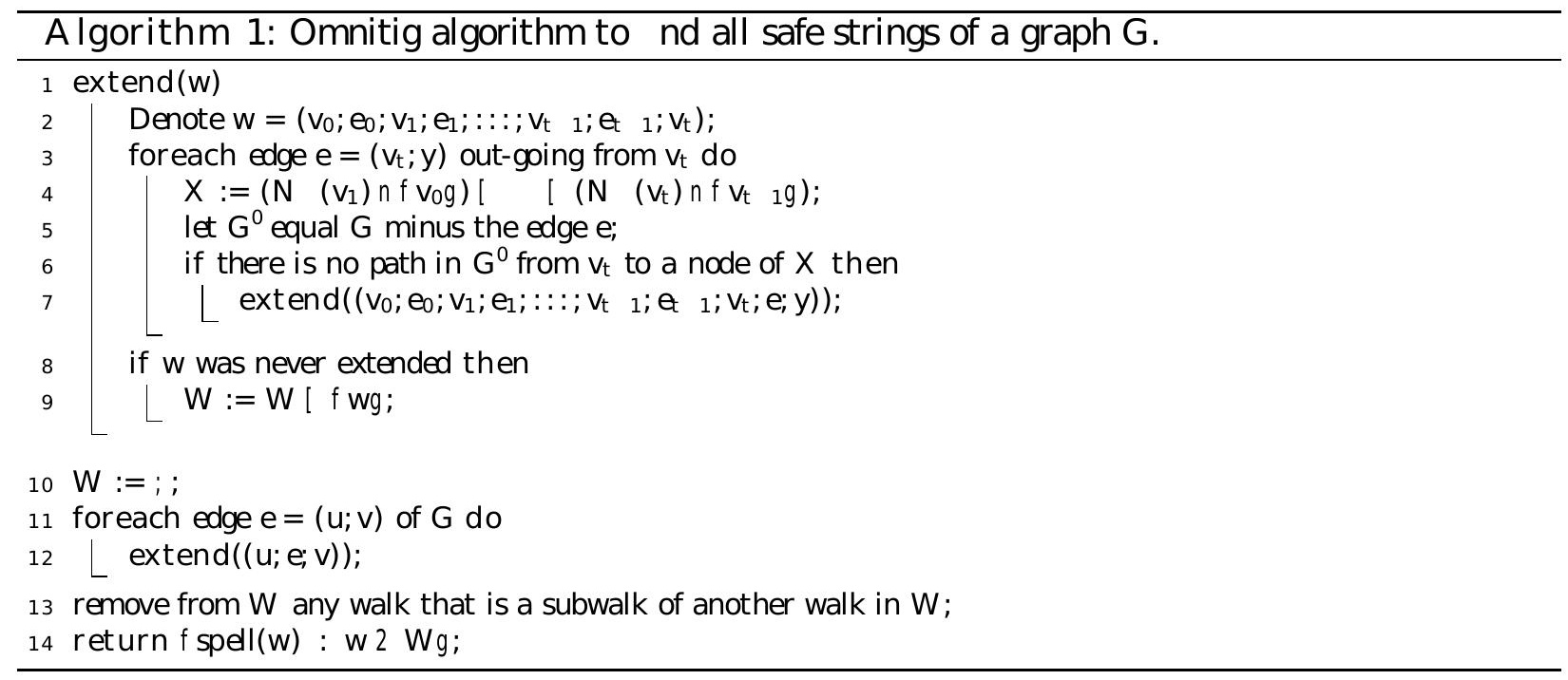

Since $A$ is circular and covers all edges of $G$, then after traversing $w_{j}$, the walk $A$ eventually visits the edge $e_{\text {. }}$. The walk $A$ may visit $v_{j}$ multiple times before traversing the edge $e_{j}$. Let $w^{0}$ denote the subwalk of $A$ between the last two occurrences of $v_{j}$ before $A$ traverses the edge $\mathrm{e}$. Since $w^{0}$ is a proper $v_{j}-v_{j}$ walk, $16 j 6 t$, and $w$ satis es (3), we have that one of the following must hold:

the walk $\left(v_{j} ; e_{i} ;:: ; v_{t} ; e_{i} ; v_{t+1}\right)$ is a pre $x$ of $w^{0}$. this contradicts the fact that $w^{0}$ is a subwalk of $A$ between $v_{j}$ and the immediately next occurrence of e (since in this case $w^{0}$ contains $e_{\text {) }}$ ); the walk $\left(v_{0} ; e_{0} ;:: ; v_{j} 1 ; e_{1} 1 ; v_{j}\right)$ is a su $x$ of $w^{0}$. this implies that $\left(w^{0}, e_{j} ; v_{j+1}\right)$ is a longer pre $x$ of $w$ which is a subwalk of $A$, contradicting the maximality of $w_{j}$;

the walk $w$ appears on $w^{0}$. since $w^{0}$ is a subwalk of $A$, this implies that also $w$ is a subwalk of $A$, contradicting again the maximality of $w_{j}$.

\section{Omnitig algorithm}

In this section, we use Theorem 1 to give the omnitig algorithm (Algorithm 1) and prove that it runs in polynomial time (Theorem 2). The al gorithm nds all maximal omnitigs of $G(R)$, which, by Theorem 1, are exactly the maximal safe strings of $G(R)$. Our al gorithm is based on the following observation, which follows directly from the de nition of omnitigs:

Observation 1. Consider a walk $w^{0}=\left(v_{0} ; e_{0} ;:: ; \Theta_{t} ; ; v_{t} ; e_{t} ; v_{t+1}\right)$ of length at least two, and consider its subwalk $w=\left(v_{0} ; e_{0} ;:: ; \theta_{e} 1 ; v_{t}\right)$. Then $w^{0}$ is an omnitig if and only if (i) $w$ is an omnitig and (ii) for all 06 i $6 \mathrm{t} \quad 1$, there is no proper $v_{t}-v_{i}$ path with rst edge di erent from $e$ and last edge di erent from $e_{1}$.

The idea of the algorithm is to start an exhaustive traversal of $\mathrm{G}$ from every edge (Lines 11-12), which by de nition is an omnitig, and to keep traversing edges as long as the current walk is an omnitig. An omnitig w is thus recursively constructed, by possibly extending to the right with each 
edge e out-going from its last vertex (Lines 3-7). If w extended with e is not an omnitig, then we abandon this extension because Observation 1 tells us that no further extension could be an omnitig. To dheck if this extension is an omnitig or not, it is enough to check whether condition (ii) of Observation 1 is satis ed. Condition (i) is automatically satis ed because of the structure of the algorithm \{we extend only walks that are omnitigs. The omnitigs found are saved in a set W (Line 9), except for those omnitigs that are obviously non-maximal (Line 8). In the nal step (Lines 13-14), we remove the non-maximal omnitigs from $W$ and report the rest.

To check that condition (ii) is satis ed (Lines 4-6), we take the set X (Line 4) and check if there is a path starting with an edge out-going from $v_{t}$ and di erent from $e$, and leading to a node of $X$. The correctness of this procedure can be seen as follows. If there is no such path, then we know that there is no path satisfying (ii). If we do nd a path $p$ from $v_{t}$ to some in-neighbor $x 2 \times$ of some $v_{i}$, and $p$ does not use $v_{i}$, then the path obtained by extending $p$ to $v_{i}$ contradicts (ii). If $p$ contains $v_{i}$, then such an extension is not possible, because a path cannot repeat a vertex; however, we will show that $p$ cannot use $v_{i}$ by contradiction. Assume that it does, and observe that after passing through $v_{i}$, the path $p$ cannot pass again through $v_{t}$. Let $v_{j}, i \quad 6 j<t$, be the rst vertex that $p$ visits after $v_{i}$ such that from $v_{j}$ it continues with an edge $e^{0} G$ e. Let $p^{0}$ denote the $v_{j}-x$ subpath of $p$ from $v_{j}$ until $x$. We obtained that $p^{0}$ followed by $v_{i}$ is a proper $v_{j}-v_{i}$ path with rst

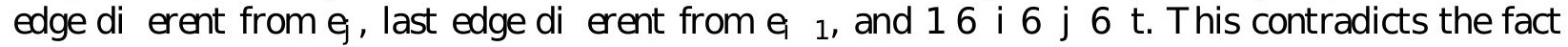
that $w$ (the walk we are extending) is an omnitig.

Next, we show that the algorithm runs in polynomial time. First, we show that the number of omnitigs included in $\mathrm{W}$ and their length, prior to removal of non-maximal ones, is polynomial:

Lemma 1. Let $W$ be a set of omnitigs in an edge-centric graph mode $G(R)$, whose genome graph is di erent than a single cycle. Furthermore, suppose no omnitig in $\mathrm{W}$ is a pre $\mathrm{x}$ of another omnitig in W. Then, jWj $6 \mathrm{~nm}$ and the length of any omnitig in $\mathrm{W}$ is $\mathrm{O}(\mathrm{nm})$.

Proof. We rst show that we can visit the edges of $G=G(R)$ with a circular edge-covering walk $\mathrm{C}$ of at most $\mathrm{nm}$ nodes. Let $\mathrm{e}_{0} ;::: ; \mathrm{e}_{\mathrm{m}} 1$ be an arbitrary order of the edges of $\mathrm{G}$. Since we assume that $\mathrm{G}$ admits one genomic reconstruction, then $\mathrm{G}$ is strongly connected. Thus, from every end extremity of $e_{i}$ there is a path to the start extremity of $e_{(i+1) \operatorname{mod~m}}, 06$ i $6 \mathrm{~m} \quad 1$, of length at most $n$ 1. Therefore, $C$ can be constructed to rst visit $e_{0}$, then to follow such a path until $e_{1}$, and so on until $e_{m}$, from where it follows such a path back to $e_{0}$.

By Theorem 1, we have that any omnitig of $G$ is a subwalk of $C$. We can associate every $w 2 \mathrm{~W}$ with all the start positions in $\mathrm{C}$ (in terms of nodes) where it is a subwalk. Because W does not contain walks that are pre xes of other walks, a position of $C$ can have at most one walk associated with it. Since jCj $6 \mathrm{~nm}, \mathrm{~W}$ can contain at most nm walks.

It remains to prove that the length of any omnitig in $\mathrm{W}$ is $\mathrm{O}(\mathrm{nm})$. To simplify notation, rename $C$ as $\left(v_{0} ; e_{0} ; v_{1} ; e_{1} ;:: ; v_{t} ; e_{i} ; v_{t+1}\right)$ with $v_{t+1}=v_{0}$. Since $G$ is di erent than a single cycle, then there exist $v_{j}$ and $v_{i}$ on $C$, such that $e=\left(v_{j} ; v_{i}\right)$ is an edge of $G$, and e $z f e ; e$ ig. Any omnitig (thus a subwalk of $C$ ) cannot contain twice $v_{j}$ and $v_{i}$ as internal nodes, since otherwise the proper path $\left(v_{j} ; e ; v_{i}\right)$ violates the omnitig de nition. Thus the length of any omnitig is $\mathrm{O}(\mathrm{nm})$.

(As an aside, it remains open whether the bound on $\mathrm{jWj}$ can be reduced to $\mathrm{m}$, which is the case for unitigs; our experiments from Section 8 suggest this may be the case in practice.) Note that Line 8 guarantees that $W$, prior to removal of subwalks in Line 13, satis es the pre $x$ condition of Lemma 1. Lemma 1 then implies that reporting one omnitig by our algorithm takes polynomial 
time, and there are only polynomially many omnitigs reported. Furthermore, removing the nonmaximal omnitigs (Line 13) can be done in linear time in the sum of the omnitig lengths, by appropriately traversing a su $x$ tree constructed from them. Thus, we have our main theorem:

Theorem 2. Let $R$ be a set of reads and let $G(R)$ be an edge-centric graph model. Algorithm 1 outputs in polynomial time all safe strings of $G(R)$.

Finally, we note some implementation details that are crucial in practice Prior to starting, we apply the $\mathrm{Y}$-to-V algorithm and the standard graph compaction algorithm to compact unitigs [6]. This signi cantly reduces the number of nodes/ edges in the graph without changing the maximal safe strings. We also precompute all omnitigs of length two and store them in a hash table, so that whenever we want to extend the omnitig w in Line 6, we check beforehand whether the pair (et 1 ; e) is stored in the hash table. This signi cantly limits, in practice, the number of graph traversals we have to do at Line 6. Finally, we do not compute the set $X$ every time, but instead incrementally built it up as we extend the omnitig w. Our implementation is freely available for use

\section{Node-centric model}

In this section we obtain analogous results for nodecentric modes, though both the de nitions, algorithms, and proofs need to modi ed. The following de nition is similar to the one for the edge-centric mode, the only addition being its second bullet (see Figure 5 for an illustration).

De nition 6 (Omnitig, node-centric model). Let $G$ be a directed graph and let $w=$ $\left(v_{0} ; e_{0} ; v_{1} ; e_{1} ;::: ; v_{t} ; e_{t} ; v_{t+1}\right)$ be a walk in $G$. We say that $w$ is a omnitig $i$ the following two conditions hold:

for all 16 i 6 j $6 \mathrm{t}$, there is no proper $\mathrm{v}_{\mathrm{j}}-\mathrm{v}_{\mathrm{i}}$ path with rst arc di erent from $\mathrm{e}$, and last arc di erent from $\mathrm{e}_{\mathrm{i}} 1$.

for all $06 \mathrm{j} 6 \mathrm{t}$, the arc $\mathrm{e}$ is the only $v_{j}-v_{j+1}$ path.

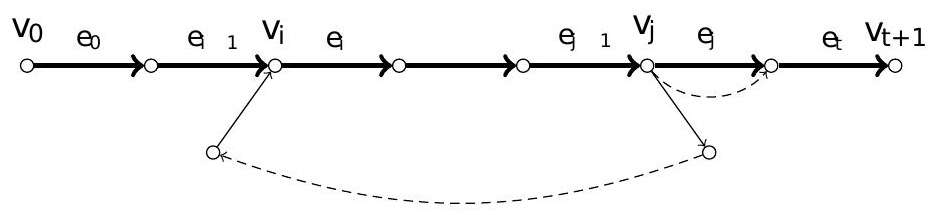

Fig. 5. An illustration of the omnitig de nition, node-centric mode

The following theorem is analogous to Theorem [1, and characterizes the safe strings in the nodecentric model.

Theorem 3. Given a node-centric graph model $G=G(R)$ built for a set of reads $R$, and a string $\mathrm{S}$, the following three statements are equivalent:

(1) $\mathrm{S}$ is a safe string for $\mathrm{G}$;

\footnotetext{
${ }^{1}$ htt ps: // gi thub. conhal exandrut onescu/ conpl et e- cont i gs
} 
(2) $\mathrm{s}$ is spelled by a walk $w=\left(v_{0} ; e_{0} ; v_{1} ; e_{1}::: ; v_{t} ; e_{t} ; v_{t+1}\right)$ in $G$ and $w$ is a omnitig;

(3) $s$ is spelled by a walk $w=\left(v_{0} ; e_{0} ; v_{1} ; e_{1}::: ; v_{t} ; e_{i} ; v_{t+1}\right)$ in $G$ and $w$ satis es: for all $06 \mathrm{j} 6 \mathrm{t}$, the arc $e_{j}$ of $w$ is the only $v_{j}-v_{j+1}$ path, and for all $16 j 6 t$, all proper $v_{j}-v_{j}$ (circular) walks $\mathrm{w}^{0}$ ful $\mathrm{II}$ at least one of the following conditions:

(i) the subwalk $\left(v_{j} ; e ;::: ; v_{t} ; e_{t} ; v_{t+1}\right)$ of $w$ is a pre $x w^{0}$, or

(ii) the subwalk $\left(v_{0} ; e_{0} ;::: ; v_{j} 1 ; e_{1} 1 ; v_{j}\right)$ of $w$ is a su $x$ of $w^{0}$, or

(iii) $\mathrm{w}$ is a subwalk of $\mathrm{w}^{0}$.

We analogously prove Theorem 3 by proving the cyclical sequence of implications (1) ) (2) ) (3) ) (1).

Proof of (1) ) (2). Assume that $s$ is a safe string for R. By de nition of a graph model, a safe string for $\mathrm{R}$ is spelled by a unique walk in a node-centric model for $\mathrm{R}$. Let this walk be $\mathrm{w}$, and let A be a circular node-covering walk of $G$ (thus containing $w$ as subwalk).

First, assume for a contradiction that there exist 16 i 6 j $6 \mathrm{t}$, and a proper $\mathrm{v}_{\mathrm{j}}-\mathrm{v}_{\mathrm{i}}$ path $\mathrm{p}$ whose rst arc is di erent from $\mathrm{e}$ and its last arc is di erent from e 1 . From $A$, we can construct another circular node-covering walk $B$ of $G$ which does not contain w as subwalk, and thus spell(B) does not contain $\mathrm{s}$ as sub-string. This will contradict the fact that $\mathrm{s}$ is a safe string for $\mathrm{G}$.

Whenever $A$ visits node $v_{j}$, then $B$ follows the $v_{j}-v_{i}$ path $p$, then it follows $\left(v_{i} ; e_{i} ;:: ; e_{j} 1 ; v_{j}\right)$, and nally continues as $A$. To see that $w$ does not appear as a subwalk of $B$, consider the subwalk $w^{0}=\left(v_{i} 1 ; e_{1} 1 ; v_{i} ; e_{i} ;:: ; e_{j} 1 ; v_{j} ; e_{j} ; v_{j+1}\right)$ of $w$ (recall that 16 i $\left.6 \mathrm{j} 6 \mathrm{t}\right)$. Since $p$ is proper, and its rst arc is di erent from $e_{j}$ and its last arc is di erent from e ${ }_{1}$, then, by construction, the only way that $w^{0}$ can appear in $B$ is as a subwalk of $p$. However, this implies that both $v_{j}$ and $v_{i}$ appear twice on $p$, contradicting the fact that $p$ is $a v_{j}-v_{i}$ path.

Second, assume for a contradiction that there is some $06 \mathrm{j} 6 \mathrm{t}$ and another $v_{j}-v_{j+1}$ path $p^{0}$ than the arc $e_{\text {. }}$ J ust as above, from A we can construct another node-covering walk $C$ that avoids w (and thus spell(C) does not contain $\mathrm{S}$ as sub-string) as follows. Whenever A traverses the arc $\mathrm{e}, \mathrm{C}$ traverses instead $\mathrm{p}^{0}$. The walk $C$ is node-covering because it still covers all nodes of $w$, and otherwise $C$ coincides with $A$. However, it does not contain $w$ as subwalk because $p^{0}$ is di erent from the arc $\mathrm{e}$, and, since it is a path, it cannot pass through e again, as otherwise it would visit twice either $v_{j}, v_{j}+1$, or both.

The proof of (2) ) (3) is identical to the corresponding proof of (2) ) (3) for Theorem1.

Proof of (3) ) (1). Assume w satis es (3), and let A be a circular nodecovering walk of $G$. We need to show that $w$ is a subwalk of $A$. Let $w_{j}=\left(v_{0} ; e_{0} ;:: ; ; v_{j} 1 ; e_{1} ; v_{j}\right)$ be the longest pre $x$ of $w$ that $A$ ever traverses, ending at some $v_{j}$. Since $A$ covers all nodes, then $j>0$. Suppose for a contradiction that $\mathrm{j} \in \mathrm{t}+1$. Since $A$ is circular and covers all nodes of $G$, then after traversing $w_{j}$, the walk $A$ eventually visits the node $v_{j+1}$. The walk $A$ may, or may not, visit $v_{j}$ again before visiting the node $v_{j}+1$.

First, suppose that after visiting $v_{j}$ at the end of $w_{j}, A$ visits again $v_{j}$ before visiting $v_{j}+1$. Let $w^{0}$ denote the subwalk of $A$ between the last two occurrences of $v_{j}$ before visiting the node $v_{j+1}$. If $16 \mathrm{j} 6 \mathrm{t}$, since $\mathrm{w}^{0}$ is a $\mathrm{v}_{\mathrm{j}}-\mathrm{v}_{\mathrm{j}}$ walk, and $\mathrm{w}$ satis es (3), we have that either:

the walk $\left(v_{j} ; e_{i} ; v_{j+1} ;::: ; v_{t} ; e_{t} ; v_{t+1}\right)$ is a pre $x$ of $w^{0}$. this contradicts the fact that $w^{0}$ is a subwalk of $A$ between $v_{j}$ and the immediately next occurrence of $v_{j+1}$, since in this case $w^{0}$ would contain $v_{j+1}$ more times; 
the walk $\left(v_{0} ; e_{0} ;::: ; v_{j} 1 ; e_{1} 1 ; v_{j}\right)$ is a su $x$ of $w^{0}$. this implies that $\left(w^{0}, e ; v_{j+1}\right)$ is a longer pre $x$ of $w$ that is a subwalk of $A$, contradicting the maximality of $w_{j}$;

the walk $w$ appears on $w^{0}$. since $w^{0}$ is a subwalk of $A$, this implies that also $w$ is a subwalk of $A$, contradicting again the maximality of $w_{j}$.

If $j=0$, then by removing all cycles from $w^{0}$, we obtain $a v_{j}-v_{j+1}$ path, di erent than the arc $e_{0}$, since otherwise we would contradict the maximality of $w_{j}$. But this contradicts the fact that $w$ is satis es (3).

Second, suppose that the walk $A$ does not visit $v_{j}$ again after $w_{j}$ and before visiting $v_{j+1}$. Let $w^{\infty}$ be the $v_{j}-v_{j+1}$ subwalk of $A$ between $w_{j}$ and this next occurrence of $v_{j+1}$. The walk $w^{\infty}$ may not be a path, but by removing all cycles from it we obtain a $v_{j}-v_{j}+1$ path $w^{\infty}$. This path is di erent from $e$ by the maximality of $w_{j}$, contradicting again the fact that $w$ satis es (3).

A nalogous to the edgecentric case, we can prove the following polynomial upper-bound on the number and length of all omnitigs.

Lemma 2. Let $W$ be a set of omnitigs in a node-centric graph model $G(R)$, whose genome graph is di erent than a single cycle. Furthermore, suppose no omnitig in $W$ is a pre $x$ of another omnitig in W. Then, jWj $6 \mathrm{n}^{2}$ and the length of any omnitig in $\mathrm{W}$ is $\mathrm{O}\left(\mathrm{n}^{2}\right)$.

We also leave open the question whether the bound on the number of maximal omnitigs in the nodecentric model can be reduced to $\mathrm{n}$. We now combine Theorem 3 and Lemma 2 for obtaining our polynomial-time safe and complete assembly algorithm.

Theorem 4. There is a safe and complete assembly algorithm for any node-centric graph model $G(R)$ built on a set $R$ of reads, which runs in polynomial time.

Proof. The proof is identical to the one for the edgecentric case (Algorithm 1 and Theorem 2). The only di erence that needs to be made to Algorithm[1] is to check that the second bullet in the de nition of omnitig for the nodecentric case holds. This can be similarly performed by a single graph traversal, and only for the last edge added to the omnitig.

\section{Experimental results}

We wanted to test the potential of omnitigs as an alternative to unitigs, under the assumptions of Section 4. We chose two genomes: one bacterial genome, E.coli, and one larger genome, Human chr10 (circularized). The graph model was the edge-centric de Bruijn graph built on the set of all $(k+1)$-mers of the genome. We used $k=31$ and $k=55$ for E. coli and chr10, respectively, according to what has been used in practice for the assembly of such genomes.

We wanted to measure the e ect of omnitigs on assembly contiguity in terms of (1) increase in contig length, and (2) increase of biological context for elements of interest. To measure the increase in length, we measured the average contig length and the E-size. Since multiple contigs can cover overlapping regions, we found the E-size metric [36] to be more appropriate than the N50 metric. The E-size of a set of substrings of a genome is de ned as the average, over all genomic positions $i$, of the mean length of all substrings spanning position $i$. This was computed by aligning the contigs to the reference. Table]1 shows that omnitigs exhibit signi cantly more contiguity than unitigs, with an average contig length that is $62-82 \%$ higher. There is very little improvement in the 
Table 1. Results for $D B_{e c}^{k}(R)$, where $R$ is the set of all $(k+1)$-mers of the genome.

\begin{tabular}{|l|r|r|r|r|r|r|r|r|}
\hline & \multicolumn{3}{|c|}{ E.coli (k=31) } & \multicolumn{3}{c|}{ chr10 (k 55) } \\
\hline & \# strings & avg len & E-size & time (s) & \# strings & avg len & E-size & time (s) \\
\hline \hline unitigs & 1,743 & 2,654 & 33,309 & $<1$ & 259,845 & 546 & 8,344 & 1 \\
Y-to-V & 1,004 & 4,682 & 33,632 & $<1$ & 159,101 & 878 & 8,376 & 2 \\
omnitigs & 983 & 4,832 & 34,557 & $<1$ & 158,236 & 887 & 8,401 & $1 ; 046$ \\
\hline
\end{tabular}

Fig. 6. The increase in SNP block size in omnitigs compared to unitigs (A) and $Y$-to-V contigs (B). Each point is a SNP, and the $x$-value is the block size of the unitig (in A) or $Y$-to-V contig (in B) covering it. The $y$-value is the increase in the block size, when compared with omnitigs. Note that the y-axis does not represent the block size, but a di erence of block sizes.

E-size (1-4\%), indicating that most of the improvement come from increasing the length of shorter contigs.

We wanted to also measure the potential of omnitigs to improve downstream biological analysis, relative to unitigs. Longer contigs can provide more anking context around important genomic elements such as SNPs. One general type of study collects statistics about the relationship of each SNP to other SNPs on the same contig; such a study is necessarily limited by the number of SNPs present on the same contig [39]. We call this number the block size of a SNP. To see the e ect of omnitigs on such a study, we identi ed chr10 locations of SNPs in the human population (using dbSNP), and the block size of each SNP in the omnitig vs. the unitig algorithms. Figure $6 \mathrm{~A}$ shows that omnitigs in many cases provide more SNP context. The number of SNPs whose block size increased was 1:7 million (out of 5:9 million) and whose block size increased by more than 10 was 137 thousand. The average number of SNPs per omnitig was 41, with only 26 per unitig. Consistent with the contiguity results of Table 1 , the e ect is more pronounced on contigs with less SNPs on them. 
We also compared omnitigs to $Y$-to-V contigs. $Y$-to-V contigs have been proposed in the literature [22 12 17], but, to the best of our knowledge, there has not been a quantitative study comparing their merits against other contig al gorithms. Omnitigs also provide more SNP context than Y-to-V contigs, with 266 thousand SNPs having an increase in block size (Figure 6B). Omnitigs are only marginally better than $Y$-to- $V$ contigs in terms of average contiguity (Table 1). Our results suggest that, though not as bene cial as omnitigs, $Y$-to- $V$ contigs may nevertheless providea better alternative to unitigs that is faster than the omnitig algorithm.

Table 1 also shows the wall-clock running times of our algorithms. The experiments were run on a node with two Xeon $2.53 \mathrm{GHz}$ CPUs. We parallelized the omnitig algorithm so that it utilized all 8 available cores. We observe negligible running times for all algorithms on E.coli. On chr10, the running time of the omnitig algorithm is signi cantly longer (by 18mins) than the unitig or $Y$-to-V algorithm, though it would still not form a bottleneck in an assembly pipeline. The memory usage did not exceed 1 GB at any point, though we believe it can be signi cantly reduced with a more careful implementation.

\section{Conclusion}

Therearetwo natural directions for future work: practical and theoretical. In the practical direction, the omnitig al gorithm should be extended to handle the complexities of real data such as sequencing errors, imperfect coverage, linear genomes, and doublestrandedness. This is a non-trivial task which is outside the scope of the current study, but it will be important in facilitating the application to genome analysis and assembly. In the theoretical direction, we believe that omnitigs exhibit more structure that can be exploited in a faster algorithm for nding all maximal omnitigs. We are also currently studying the graph model which a genomic reconstruction is any collection of circular walks that together cover all nodes/ edges of the graph (as in metagenomic sequencing of bacteria). We are also studying the dass of genome graphs admitting a single safe walk covering all of their nodes or edges, question related to the ones about unique reconstructions.

\section{Acknowledgments}

We would like to thank Daniel Lokshtanov for initial discussions, Rayan Chikhi for feedback on the manuscript, and Nidia Obscura Acosta for very helpful discussion on the manuscript. This work was supported in part by NSF awards DBI-1356529, IIS-1453527, and IIS-1421908 to PM, and by A cademy of Finland grant 274977 to AT.

\section{A uthor Disclosure Statement}

No competing nancial interests exist.

\section{References}

1. Bankevich, A., Nurk, S., Antipov, D., Gurevich, A.A., Dvorkin, M., Kulikov, A.S., Lesin, V.M., Nikolenko, S.I., Pham, S.K., Prjibelski, A.D., Pyshkin, A., Sirotkin, A., Vyahhi, N., Tesler, G., Alekseyev, M.A., Pevzner, P.A.: SPAdes: A new genome assembly algorithm and its applications to singlecell sequencing. J ournal of Computational Biology 19(5) (2012) 455\{477 
2. Boetzer, M., Henkel, C.V., J ansen, H.J ., Butler, D., Pirovano, W.: Sca olding pre-assembled contigs using SSPACE. Bioinformatics 27(4) (2011) 578\{579

3. Boetzer, M., Pirovano, W.: Toward almost closed genomes with gap ller. Genome Biology 13(6) (2012) $1\{9$

4. Boisvert, S., Laviolette, F., Corbeil, J.: Ray: simultaneous assembly of reads from a mix of high-throughput sequencing technologies. J ournal of Computational Biology 17(11) (2010) 1519\{1533

5. Bresler, G., Bresler, M., Tse, D.: Optimal Assembly for High Throughput Shotgun Sequencing. BMC Bioinformatics 14(Suppl 5) (2013) S18

6. Chikhi, R., Limasset, A., J ackman, S., Simpson, J .T., Medvedev, P.: On the representation of de bruijn graphs. In: Research in Computational Molecular Biology, Springer (2014) 35\{55

7. Chikhi, R., Rizk, G.: Spacee cient and exact de Bruijn graph representation based on a Bloom Iter. In: WABI. Volume 7534 of Lecture Notes in Computer Science, Springer (2012) $236\{248$

8. Guenoche, A.: Can we recover a sequence, just knowing all its subsequences of given length? Computer Applications in the Biosciences 8(6) (1992) $569\{574$

9. Haussler, D., O'Brien, S.J ., Ryder, O.A., Barker, F.K., Clamp, M., Crawford, A.J ., Hanner, R., Hanotte, O., J ohnson, W.E., McGuire, J .A., et al.: Genome 10K: a proposal to obtain wholegenome sequence for 10,000 vertebrate species. J ournal of Heredity 100(6) (2008) $659\{674$

10. Idury, R.M., Waterman, M.S.: A new algorithm for DNA sequence assembly. J ournal of computational biology 2(2) (1995) $291\{306$

11. Iu, Florent'ev, V.L., Khorlin, A.A., Khrapko, K.R., Shik, V.V.: Determination of the nucleotide sequence of DNA using hybridization with oligonucleotides. A new method. Doklady Akademii nauk SSSR 303(6) (1988) $1508\{1511$

12. J ackson, B.G.: Parallel methods for short read assembly. PhD thesis, lowa State University (2009)

13. Kapun, E., Tsarev, F.: De Bruijn superwalk with multiplicities problem is NP-hard. BMC bioinformatics 14(Suppl 5) (2013) S7

14. Kapun, E., Tsarev, F.: On NP-hardness of the paired de Bruijn sound cycle problem. In: Algorithms in Bioinformatics. Springer (2013) $59\{69$

15. Kececioglu, J.D., Myers, E.W.: Combinatiorial algorithms for DNA sequence assembly. Algorithmica 13(1/2) (1995) $7\{51$

16. Kececioglu, J .D.: Exact and approximation algorithms for DNA sequence reconstruction. PhD thesis, University of Arizona, Tucson, AZ, USA (1992)

17. Kingsford, C., Schatz, M.C., Pop, M.: Assembly complexity of prokaryotic genomes using short reads. BMC bioinformatics 11(1) (2010) 21

18. Lam, K., Khalak, A., Tse, D.: Near-optimal assembly for shotgun sequencing with noisy reads. BMC Bioinformatics 15(S-9) (2014) S4

19. Lander, E.S., Waterman, M.S.: Genomic mapping by ngerprinting random clones: a mathematical analysis. Genomics 2(3) (1988) 231\{239

20. Luo, R., Liu, B., Xie, Y., Li, Z., Huang, W., Yuan, J ., He, G., Chen, Y., Pan, Q., Liu, Y., et al.: Soapdenovo2: an empirically improved memory-e cient short-read de novo assembler. GigaScience 1(1) (2012) 18

21. Medvedev, P., Brudno, M.: Maximum likelihood genome assembly. J ournal of computational biology 16(8) (2009) $1101\{1116$

22. Medvedev, P., Georgiou, K., Myers, G., Brudno, M.: Computability of models for sequence assembly. In: WABI. (2007) $289\{301$

23. Medvedev, P., Pham, S., Chaisson, M., Tesler, G., Pevzner, P.: Paired de Bruijn graphs: a novel approach for incorporating mate pair information into genome assemblers. J ournal of Computational Biology 18(11) (2011) $1625\{1634$

24. Miller, J.R., Koren, S., Sutton, G.: Assembly algorithms for next-generation sequencing data. Genomics 95(6) (2010) $315\{327$

25. Motahari, A.S., Bresler, G., Tse, D.N.C.: Information theory of DNA shotgun sequencing. IEEE Transactions on Information Theory 59(10) (2013) 6273\{6289

26. Myers, E.W.: The fragment assembly string graph. In: ECCB/J BI. (2005) 85

27. Nagarajan, N., Pop, M.: Parametric complexity of sequence assembly: Theory and applications to next generation sequencing. J ournal of Computational Biology 16(7) (2009) 897\{908

28. Nagarajan, N., Pop, M.: Sequence assembly demysti ed. Nature Reviews Genetics 14(3) (2013) $157\{167$

29. Narzisi, G., Mishra, B., Schatz, M.C.: On algorithmic complexity of biomolecular sequence assembly problem. In: Algorithms for Computational Biology. Springer (2014) 183\{195

30. Peltola, H., Soderlund, H., Tarhio, J., Ukkonen, E.: Algorithms for some string matching problems arising in molecular genetics. In: IFIP Congress. (1983) $59\{64$ 
31. Pevzner, P.A.: L-Tuple DNA sequencing: computer analysis. J Biomol Struct Dyn 7(1) (Aug 1989) $63\{73$

32. Pevzner, P.A., Tang, H., Waterman, M.S.: An Eulerian path approach to DNA fragment assembly. Proceedings of the National Academy of Sciences 98(17) (2001) 9748\{9753

33. Rubinov, A.R., Gelfand, M.S.: Reconstruction of a string from substring precedence data. J ournal of Computational Biology 2(2) (1995) 371\{381

34. Sahlin, K., Vezzi, F., Nystedt, B., Lundeberg, J ., Arvestad, L.: BESST-e cient sca olding of large fragmented assemblies. BMC bioinformatics 15(1) (2014) 281

35. Salmela, L., Sahlin, K., Makinen, V., Tomescu, A.I.: Gap Iling as exact path length problem. In: Research in Computational Molecular Biology, Springer (2015) $281\{292$

36. Salzberg, S.L., Phillippy, A.M., Zimin, A., Puiu, D., Magoc, T., Koren, S., Treangen, T.J ., Schatz, M.C., Delcher, A.L., Roberts, M.: GAGE: a critical evaluation of genome assemblies and assembly al gorithms. Genome Research (2011)

37. Simpson, J .T ., Durbin, R.: E cient construction of an assembly string graph using the FM-index. Bioinformatics 26(12) (2010) i $367\{i 373$

38. Simpson, J .T ., Durbin, R.: E cient de novo assembly of large genomes using compressed data structures. Genome Research (2011)

39. Uricaru, R., Rizk, G., Lacroix, V., Quillery, E., Plantard, O., Chikhi, R., Lemaitre, C., Peterlongo, P.: Referencefree detection of isolated SNPs. Nucleic Acids Research 43(2) (2015) ell

40. Vyahhi, N., Pyshkin, A., Pham, S., Pevzner, P.A.: From de Bruijn graphs to rectangle graphs for genome assembly. In: Algorithms in Bioinformatics. Springer (2012) 249\{261

41. Waterman, M.S.: Introduction to computational biology: maps, sequences and genomes. CRC Press (1995)

42. Zerbino, D.R., Birney, E.: Velvet: algorithms for de novo short read assembly using de Bruijn graphs. Genome Research 18(5) (2008) $821\{829$ 